\title{
FINITENESS OF RATIONAL CURVES OF DEGREE 12 ON A GENERAL QUINTIC THREEFOLD
}

\author{
EDOARDO BALLICO AND CLAUDIO FONTANARI
}

\begin{abstract}
We prove the following statement, predicted by Clemens' conjecture: A generic quintic threefold contains only finitely many smooth rational curves of degree 12 .
\end{abstract}

\section{INTRODUCTION}

The present paper is entirely devoted to the proof of the following instance of Clemens' conjecture (4

Theorem 1. A generic quintic threefold contains only finitely many smooth rational curves of degree 12 .

We point out that the cases $d \leq 11$ have been previously addressed in 14 $(d \leq 7)$, 17] and [13] $(d=8,9),[5](d=10),[6]$ and $[7](d=11)$, and we recall the general set-up.

Let $M_{d}$ be the set of smooth rational curves of degree $d$ in $\mathbb{P}^{4}$. It is smooth and irreducible of dimension $5 d+1$. Let $\mathbb{P}^{125}$ denote the projective space of all quintic hypersurfaces of $\mathbb{P}^{4}$ and consider the incidence correspondence $I_{d}=\{(C, W): C \subset$ $W\} \subset M_{d} \times \mathbb{P}^{125}$. Let $\pi_{1}: I_{d} \rightarrow M_{d}$ and $\pi_{2}: I_{d} \rightarrow \mathbb{P}^{125}$ denote the restrictions to $I_{d}$ of the two projections.

The map $\pi_{2}$ turns out to be finite if for every irreducible family $\Gamma \subseteq M_{d}$ with general element $C$ we have:

$$
\operatorname{dim} \Gamma+\left(h^{0}\left(\mathcal{I}_{C}(5)\right)-1\right) \leq 125 .
$$

From the standard exact sequence

$$
0 \rightarrow H^{0}\left(\mathcal{I}_{C}(5)\right) \rightarrow H^{0}\left(\mathcal{O}_{\mathbb{P}^{4}}(5)\right) \rightarrow H^{0}\left(\mathcal{O}_{C}(5)\right) \rightarrow H^{1}\left(\mathcal{I}_{C}(5)\right) \rightarrow 0
$$

it follows that

$$
h^{0}\left(\mathcal{I}_{C}(5)\right)-1=125-(5 d+1)+h^{1}\left(\mathcal{I}_{C}(5)\right)
$$

and by 2 the general curve $C$ in $M_{d}$ satisfies $h^{1}\left(\mathcal{I}_{C}(5)\right)=0$, so in order to prove Clemens' conjecture one needs to control curves $C$ with $h^{1}\left(\mathcal{I}_{C}(5)\right)>0$.

In the case $d=12$, we show that if an irreducible family $\Gamma \subseteq M_{12}$ of nondegenerate curves is a potential exception to Clemens' conjecture, then its general element $C$ satisfies $h^{1}\left(\mathcal{I}_{C}(2)\right) \geq 13$. It follows that $h^{0}\left(\mathcal{I}_{C}(2)\right) \geq 3$ and this provides a contradiction (see Lemma 1).

The key point in our reduction is to obtain $h^{1}\left(\mathcal{I}_{C}(2)\right) \geq 13$ from $h^{1}\left(\mathcal{I}_{C}(5)\right)>0$. Indeed, Lemma 2 implies that $h^{1}\left(\mathcal{I}_{C}(t-1)\right) \geq 4+h^{1}\left(\mathcal{I}_{C}(t)\right)$ except in two special cases, which are identified by Lemma 3 and then excluded in Lemmas $7,8,9,13$. Finally, a careful analysis of the degenerate case is provided (see Section 3). 
We remark that the strong form of Clemens' conjecture (as proved by Cotterill in [5] and [6] for $d=10,11$, characterizing also singular irreducible rational curves on the general quintic threefold) cannot be achieved by our methods.

We work over the complex field $\mathbb{C}$.

We thank Gilberto Bini, Gianluca Occhetta and Luis Solá Conde for helpful conversations. We are also grateful to the anonymous referee and to Carlo Madonna for useful comments.

This research was partially supported by FIRB 2012 "Moduli spaces and Applications", by GNSAGA of INdAM and by MIUR (Italy).

\section{NON-DEGENERATE CASE}

Lemma 1. If $C \in M_{12}, C$ is non-degenerate and $h^{0}\left(\mathcal{I}_{C}(2)\right) \geq 3$, then there is no smooth quintic 3-fold containing $C$.

Proof. Assume by contradiction $h^{0}\left(\mathcal{I}_{C}(2)\right) \geq 3$ and the existence of a smooth quintic 3-fold $W \subset \mathbb{P}^{4}$ with $W \supset C$ and let $E \subset \mathbb{P}^{4}$ be the intersection of 3 general element of $\left|\mathcal{I}_{C}(2)\right|$. Since $\operatorname{deg}(C)=12>8$, Bezout theorem gives the existence of an integral surface $F$ such that $C \subset F \subseteq E$. Since $C$ is non-degenerate, $F$ is non-degenerate and so $\operatorname{deg}(F) \geq 3$. Assume $E=F$, i.e. $\operatorname{deg}(F)=4$. Since the complete intersection of 2 quadric hypersurfaces is contained in exactly two linearly independent quadrics and $\operatorname{deg}(C)>8$, we get $h^{0}\left(\mathcal{I}_{C}(2)\right)=2$, a contradiction. Thus $\operatorname{deg}(F)=3$. The classification of minimal degree non-degenerate surfaces in $\mathbb{P}^{4}$ gives $h^{0}\left(\mathcal{I}_{F}(2)\right)=3$. By assumption there is $W$ with $C \subset W$. Since $\operatorname{Pic}(W)$ is freely generated by $\mathcal{O}_{W}(1), F \nsubseteq W$. Hence $W \cap F$ links $C$ to a degree 3 locally Cohen-Macaulay curve $T \subset W \cap F$. By the classification of minimal degree surfaces in $\mathbb{P}^{4}$, either $F$ is a cone with vertex o over a rational normal curve $D \subset \mathbb{P}^{3}$ or $F$ is isomorphic to the the Hirzebruch surface $F_{1}$ embedded by the complete linear system $|h+2 f|$, where $h$ is a section of the ruling of $F_{1}$ and $f$ is a fiber of the ruling of $F_{1}$.

First assume that $F$ is a cone. Since $C$ is smooth, it has multiplicity at most 1 at $o$. Hence $o \notin C$ and the linear projection from $o$ induces a degree 4 map $\ell: C \rightarrow D$. Let $\pi: G \rightarrow F$ be the blowing up of $o$ and $C^{\prime}$ the strict transform of $C$ in $G$. $G$ is isomorphic to the Hirzebruch surface $F_{3}$ and the map $\pi$ is induced by the complete linear system $|h+3 f|$. Since $o \notin C$ and $\operatorname{deg}(\ell)=4, \pi$ induces an isomorphism $C^{\prime} \rightarrow C$ and $C^{\prime} \in|4 h+12 f|$. We have $\omega_{G} \cong \mathcal{O}_{G}(-2 h-5 f)$. The adjunction formula gives $\omega_{C^{\prime}} \cong \mathcal{O}_{G}(2 h+7 f)$. Hence $h^{0}\left(\omega_{C^{\prime}}\right)>0$, contradicting the rationality and smoothness of $C^{\prime}$.

Now assume $F \cong F_{1}$. Take $a, b \in \mathbb{N}$ such that $C \in|a h+b f|$. Since $C$ is irreducible and not a line, we have $b \geq a>0$. Since $\mathcal{O}_{C}(1) \cong \mathcal{O}_{C}(h+2 f), h^{2}=-1$, $h \cdot f=1, f^{2}=0$ and $\operatorname{deg}(C)=d$, we have $12=a+b$. Since $\omega_{F_{1}} \cong \mathcal{O}_{F_{1}}(-2 h-3 f)$, the adjunction formula gives $\omega_{C} \cong \mathcal{O}_{C}((a-2) h+(b-3) f)$. Since $\operatorname{deg}\left(\omega_{C}\right)=-2$, we get $(a h+b f) \cdot((a-2) h+(b-3) f)=-2$, i.e. $-a(a-2)+a(b-3)+b(a-2)=-2$, i.e $(b-a)(a-2)+a(b-3)=-2$. Since $b \geq a>0$ and $b=12-a$, we get $a=1$ and $b=11$. Since $\mathcal{O}_{F_{1}}(5) \cong \mathcal{O}_{F_{1}}(5 h+10 f)$ and $b=11$, we have $h^{0}\left(F_{1}, \mathcal{I}_{C, F_{1}}(5)\right)=0$. Hence $W \supset F_{1}$, a contradiction.

The following fact is one of key ingredients in the proof of [7, Theorem 4.1]. 
Lemma 2. Fix integer $t \geq 2, r \geq 3$ and an integral and non-degenerate curve $T \subset \mathbb{P}^{r}$ such that $h^{1}\left(\mathcal{I}_{T}(t)\right)>0$. Assume that $h^{1}\left(M, \mathcal{I}_{M \cap T, M}(t)\right)=0$ for every hyperplane $M \subset \mathbb{P}^{r}$. Then $h^{1}\left(\mathcal{I}_{T}(t-1)\right) \geq r+h^{1}\left(\mathcal{I}_{T}(t)\right)$.

Proof. For any hyperplane $M \subset \mathbb{P}^{r}$ we have an exact sequence

$$
0 \rightarrow \mathcal{I}_{T}(t-1) \rightarrow \mathcal{I}_{T}(t) \rightarrow \mathcal{I}_{T \cap M, M}(t) \rightarrow 0
$$

Since $h^{1}\left(M, \mathcal{I}_{T, M}(t)\right)=0$, the map $H^{1}\left(\mathcal{I}_{T}(t-1)\right) \rightarrow H^{1}\left(\mathcal{I}_{T}(t)\right)$ is surjective, hence its dual $e_{M}: H^{1}\left(\mathcal{I}_{T}(t)\right)^{\vee} \rightarrow H^{1}\left(\mathcal{I}_{T}(t-1)\right)^{\vee}$ is injective. Taking the equations of all hyperplanes we get a bilinear map map $u: H^{1}\left(\mathcal{I}_{T}(t)\right)^{\vee} \times H^{0}\left(\mathcal{O}_{\mathbb{P}^{4}}(1)\right) \rightarrow$ $H^{1}\left(\mathcal{I}_{T}(t-1)\right)^{\vee}$, which is injective with respect to the second variables, i.e. for every non-zero linear form $\ell u_{\mid H^{1}\left(\mathcal{I}_{T}(t)\right)^{\vee} \times\{\ell\}}$ is injective (it is $e_{M}$ with $M:=\{\ell=0\}$ ). Hence if $(a, \ell) \in H^{1}\left(\mathcal{I}_{T}(t)\right)^{\vee} \times H^{0}\left(\mathcal{O}_{\mathbb{P}^{4}}(1)\right)$ with $a \neq 0$ and $\ell \neq 0$, then $u(a, \ell)=$ $e_{M}(a) \neq 0$. Therefore the bilinear map $u$ is non-degenerate in each variable. Hence $h^{1}\left(\mathcal{I}_{T}(t-1)\right) \geq h^{1}\left(\mathcal{I}_{T}(t)\right)+h^{0}\left(\mathcal{O}_{\mathbb{P}^{r}}(1)\right)-1$ by the bilinear lemma.

The next Lemma 3 is perhaps the technical heart of this work. It relies on a particular case of a very strong result on 0-dimensional schemes in the plane, namely, 9, Corollaire 2] (see also [9, Remarque (i)]). We recall the statement in 9] for reader's convenience. Let $E \subset \mathbb{P}^{2}$ be a zero-dimensional scheme of degree $d$. Let $\tau:=\max \left\{n: h^{1}\left(\mathcal{I}_{E}(n)>0\right\}\right.$. Let $s$ be an integer such that $s \leq d / s$ and $\tau \geq s-3+d / s$. Then either $E$ is the complete intersection of a curve of degree $s$ and a curve of degree $d / s$ and $\tau=s-3+d / s$, or there exists $s^{\prime}$ with $0<s^{\prime}<s$ and a subscheme $E^{\prime} \subset E$ contained in a curve of degree $s^{\prime}$ such that $s^{\prime}\left(\tau+\left(5-s^{\prime}\right) / 2\right) \geq \operatorname{deg}\left(E^{\prime}\right) \geq s^{\prime}\left(\tau-s^{\prime}+3\right)$. In particular, if $\tau>d / 3$, then either we have $\tau+2$ points on a line (counted with multiplicity), or we have $2 \tau+2$ or $2 \tau+3$ points on a conic (counted with multiplicity).

For the proof of Lemma 3 we also need to introduce the notion of residual scheme. Let $M$ be a projective scheme, $A$ a closed subscheme and $D \subset M$ an effective Cartier divisor of $M$. The residual scheme $\operatorname{Res}_{D}(A)$ of $A$ with respect to $D$ is the closed subscheme of $M$ with $\mathcal{I}_{A}: \mathcal{I}_{D}$ as its ideal sheaf. We always have $\operatorname{Res}_{D}(A) \subseteq A$. If $A$ is a reduced scheme, then $\operatorname{Res}_{D}(A)$ is the union of the irreducible components of $A$ not contained in $D$. If $A$ is a zero-dimensional scheme, then $\operatorname{deg}(A)=\operatorname{deg}(A \cap D)+\operatorname{deg}\left(\operatorname{Res}_{D}(A)\right)$. For any line bundle $\mathcal{L}$ on $M$ we have an exact sequence

$$
0 \rightarrow \mathcal{I}_{\operatorname{Res}_{D}(A)} \otimes \mathcal{L}(-D) \rightarrow \mathcal{I}_{A} \otimes \mathcal{L} \rightarrow \mathcal{I}_{A \cap D, D} \otimes\left(\mathcal{L}_{\mid D}\right) \rightarrow 0
$$

Lemma 3. Fix an integer $t \geq 2$. Set $M:=\mathbb{P}^{3}$ and let $Z \subset M$ a zero-dimensional scheme spanning $M$ and with $\operatorname{deg}(Z) \leq 3 t$. We have $h^{1}\left(M, \mathcal{I}_{Z, M}(t)\right) \neq 0$ if and only if either there is a line $R \subset M$ with $\operatorname{deg}(R \cap Z) \geq t+2$ or there is a conic $D \subset M$ such that $\operatorname{deg}(D \cap Z) \geq 2 t+2$ or there is a line $L \subset M$ such that $\operatorname{deg}(Z \cap L)=t+1$ and the union of the connected components of $Z$ whose reduction is contained in $L$ has degree $\geq 2 t+2$.

Proof. Set $Z_{0}:=Z$. Let $N_{1} \subset M$ be a plane such that $e_{1}:=\operatorname{deg}\left(Z \cap N_{1}\right)$ is maximal. Set $Z_{1}:=\operatorname{Res}_{N_{1}}\left(Z_{0}\right)$. For each integer $i \geq 2$ define recursively the plane $N_{i}$, the integer $e_{i}$ and the zero-dimensional scheme $Z_{i}$ in the following way. Let $N_{i} \subset M$ be any hyperplane such that $e_{i}:=\operatorname{deg}\left(Z_{i-1} \cap N_{i}\right)$ is maximal. Set $Z_{i}:=\operatorname{Res}_{N_{i}}\left(Z_{i-1}\right)$. For each $i \geq 1$ we have an exact sequence

$$
0 \rightarrow \mathcal{I}_{Z_{i}}(t-i) \rightarrow \mathcal{I}_{Z_{i-1}}(t+1-i) \rightarrow \mathcal{I}_{Z_{i-1} \cap N_{i}, N_{i}}(t+1-i) \rightarrow 0
$$


We have $e_{i} \geq e_{i-1}$ for all $i$. Since any degree 3 subscheme of $M$ is contained in a plane, if $e_{i} \leq 2$, then $Z_{i-1} \subset N_{i}$ and $Z_{i}=\emptyset$. Since $\operatorname{deg}(Z) \leq 3 t$, there is an integer $i$ such that $1 \leq i \leq t$ and $Z_{i}=\emptyset$. From (4) we get an integer $i \in\{1, \ldots, t\}$ such that $h^{1}\left(N_{i}, \mathcal{I}_{Z_{i-1} \cap N_{i}, N_{i}}(t+1-i)\right)>0$. Indeed, the fact that $Z_{i}$ is empty for some index $1 \leq i \leq t$ forces the cohomology of the ideal sheaf of $Z_{i}$ to be that of the ambient projective plane $N_{i}$. We call $c$ the minimal integer $i$. Since $h^{1}\left(N_{c}, \mathcal{I}_{Z_{i-1} \cap N_{c}, N_{c}}(t+1-c)\right)>0$, either $\left.\operatorname{deg}\left(Z_{i-1} \cap N_{c}, N_{c}\right)\right) \geq 2(t+1-c)+2$ or there is a line $L$ with $\operatorname{deg}\left(L_{c} \cap Z_{i-1} \cap N_{c}\right) \geq t+3-c$ ([3, Lemma 34]). In particular, since $c \leq t$, we have $e_{c} \geq t+3-c$. Since the sequence $\left\{e_{i}\right\}_{i \geq 1}$ is non-increasing, we have $c e_{c} \geq c(t+3-c)$. Since $\sum_{i \geq 1} e_{i}=\operatorname{deg}(Z) \leq 3 t$, we get $c(t+3-c) \leq 3 t$. Set $\psi(x)=x(t+3-x)$. The function $\psi$ is strictly increasing if $1 \leq x \leq(t+3) / 2$ and strictly decreasing if $x>(t+3) / 2$. Since $\psi(t)=3 t$ and $\psi(3)=3 t$, we get that either $1 \leq c \leq 3$ or $c=t$.

(a) Assume $c=1$. Since $Z$ spans $M$, we have $e_{1} \leq \operatorname{deg}(Z)-1$. Since $e_{1} \leq \operatorname{deg}(Z)-1$, we have $e_{1}<3 t$. By [9, Corollaire 2] (see also [9, Remarque (i)]) either there is a line $R \subset N_{1}$ with $\operatorname{deg}(R \cap Z) \geq t+2$ or there is a conic $D \subset N_{1}$ such that $\operatorname{deg}(D \cap Z) \geq 2 t+2$.

(b) Assume $c=2$. Since $e_{1} \geq e_{2}$, we have $e_{2} \leq \operatorname{deg}(Z) / 2 \leq 3 t / 2$. Since $c=2$ and $h^{1}\left(N_{2}, \mathcal{I}_{Z_{1} \cap N_{2}, N_{2}}(t-1)\right)>0$, by [3, Lemma 34] either $e_{2} \geq 2 t$, which is a contradiction, or there is a line $R \subset N_{2}$ such that $\operatorname{deg}\left(R \cap Z_{1}\right) \geq t+1$. If $\operatorname{deg}(R \cap Z) \geq t+2$, then we are done. Hence we may assume $\operatorname{deg}(Z \cap R)=t+1$. Set $W_{0}:=Z$. Let $M_{1} \subset M$ be a plane containing $R$ and for which $f_{1}:=\operatorname{deg}\left(M_{1} \cap Z\right)$ is maximal. Since $Z$ spans $M$ we have $f_{1} \geq t+2$. Set $W_{1}:=\operatorname{Res}_{M_{1}}(Z)$. For each integer $i \geq 2$ define recursively the plane $M_{i}$, the integer $f_{i}$ and the zerodimensional scheme $W_{i}$ in the following way. Let $M_{i} \subset M$ be any hyperplane such that $f_{i}:=\operatorname{deg}\left(W_{i-1} \cap M_{i}\right)$ is maximal. Set $W_{i}:=\operatorname{Res}_{N_{i}}\left(W_{i-1}\right)$. We have $f_{i} \geq f_{i+1}$ for all $i \geq 2$, but we do not claim that $f_{1} \geq f_{2}$ (indeed, $M_{1}$ is required to contain $R$, while the $M_{i}$ with $i \geq 2$ are not). Since any degree 3 subscheme of $M$ is contained in a plane, if $f_{i} \leq 2$, then $W_{i-1} \subset M_{1}$ and $W_{i}=\emptyset$. Since $\sum_{i \geq i} f_{i}=\operatorname{deg}(Z)$ and $f_{1} \geq t+2$, we have $f_{i}=0$ for some $i<t$. Using the residual exact sequences of the planes $M_{i}$ we get the existence of a minimal integer $s \in\{1, \ldots, t-1\}$ such that $h^{1}\left(M_{s}, \mathcal{I}_{W_{s-1} \cap M_{s}, M_{s}}(t+1-s)\right)>0$. We get $f_{s} \geq t+3-s$. Since $f_{1} \geq t+2$, we get $1 \leq s \leq 2$. If $s=1$, then we use step (a) with $M_{1}$ instead of $N_{1}$. Now assume $s=2$. Since $f_{2} \leq \operatorname{deg}(Z)-f_{1} \leq 2 t-2$ and $h^{1}\left(M_{2}, \mathcal{I}_{Z_{1} \cap M_{2}, M_{2}}(t-1)\right)=0$, there is a line $L \subset M_{2}$ such that $\operatorname{deg}\left(L \cap Z_{1}\right) \geq t+1$. If $\operatorname{deg}(L \cap Z) \geq t+2$, then the lemma is true. Hence we may assume that $\operatorname{deg}(Z \cap L)=t+1$.

First assume $R \cap L=\emptyset$. Let $Q \subset M$ be a general quadric surface containing $L \cup R$. Call $\left|\mathcal{O}_{Q}(1,0)\right|$ the ruling of $Q$ containing $R$ and $L$. The residual scheme $\operatorname{Res}_{Q}(Z)$ of $Z$ has degree $\operatorname{deg}(Z)-\operatorname{deg}(Z \cap Q) \leq 3 t-(2 t+2)=t-2$ and in particular $h^{1}\left(M, \mathcal{I}_{\operatorname{Res}_{Q}(Z), M}(t-2)\right)=0$. The residual exact sequence of $Q$ gives $h^{1}\left(Q, \mathcal{I}_{Z \cap Q, Q}(t)\right) \geq h^{1}\left(M, \mathcal{I}_{Z, M}(t)\right)>0$.

Claim: We have $h^{1}\left(M, \mathcal{I}_{R \cup L}(t)\right)=0$ for every $t \geq 1$.

Proof of the Claim: Take $p \in L$. Since $R \cap L=\emptyset,\{p\} \cup R$ spans a plane, $H$. We have $(L \cup R) \cap H=R \cup\{p\}$ and hence $h^{1}\left(H, \mathcal{I}_{(L \cup R) \cap H, H}(t)\right)=0$ for all $t \geq 1$. The residual $\operatorname{Res}_{H}(L \cup R)$ of $L \cup R$ with respect to $H$ is the line $L$, because $L \cup R$ is reduced and $L \nsubseteq H$. Therefore the residual sequence of $H$ in $\mathbb{P}^{3}$ gives the following exact sequence:

$$
0 \rightarrow \mathcal{I}_{L}(t-1) \rightarrow \mathcal{I}_{L \cup R}(t) \rightarrow \mathcal{I}_{(L \cup R) \cap H, H}(t) \rightarrow 0 .
$$


Since $h^{1}\left(\mathcal{I}_{L}(t-1)\right)=0$ for all $t>0$, we get the Claim.

Since $\operatorname{deg}(Z \cap L)=\operatorname{deg}(Z \cap R)=t+1$ and $R \cap L=\emptyset$, we have $h^{1}(R \cup$ $\left.L, \mathcal{I}_{(R \cup L) \cap Z}(t)\right)=h^{1}\left(R, \mathcal{I}_{R \cap Z}(t)\right)+h^{1}\left(L, \mathcal{I}_{L \cap Z}(t)\right)=0$.

The Claim gives $h^{1}\left(M, \mathcal{I}_{Z \cap(R \cup L}(t)\right)=0$. Hence $h^{1}\left(Q, \mathcal{I}_{Z \cap(R \cup L), Q}(t)\right)=0$. The residual sequence

$$
\left.0 \rightarrow \mathcal{I}_{\operatorname{Res}_{R \cup L}(Z \cap Q)}(t-2, t)\right) \rightarrow \mathcal{I}_{Z \cap Q, Q}(t, t) \rightarrow \mathcal{I}_{(R \cup L) \cap Z, R \cup L}(t, t) \rightarrow 0
$$

gives $h^{1}\left(Q, \mathcal{I}_{\operatorname{Res}_{R \cup L}(Z \cap Q)}(t-2, t)\right)>0$. Since $\operatorname{deg}\left(\operatorname{Res}_{R \cup L}(Z \cap Q)\right)=\operatorname{deg}(Z \cap Q)-$ $2 t-2 \leq t-2$, we get a contradiction.

Now assume $R \cap L \neq \emptyset$. If $R \neq L$, then we may take the reducible conic $R \cup L$, because $R \subset M_{1}$ and $\operatorname{deg}\left(L \cap \operatorname{Res}_{M_{1}}(Z)\right)=t+1$.

Now assume $R=L$. This is the last case of the statement of the lemma.

(c) Assume $c=3$. Since $\psi(3)=3 t$, we get $e_{1}=e_{2}=e_{3}=t$. Since $e_{3}=t$ and $h^{1}\left(N_{3}, \mathcal{I}_{Z_{2} \cap N_{3}, N_{3}}(t-2)\right)>0$, there is a line $R \subset N_{3}$ such that $\operatorname{deg}\left(Z_{2} \cap R\right)=t$. Since $Z$ spans $M$, there is a plane $N^{\prime}$ such that $R \subset N^{\prime} \subset M$ and $N^{\prime} \cap Z \supsetneq Z \cap R$. Hence $e_{1} \geq \operatorname{deg}\left(N^{\prime} \cap Z\right)>t$, a contradiction.

(d) Assume $c=t$. We get $\operatorname{deg}(Z)=3 t$ and $e_{i}=3$ for all $i$. In particular $e_{1}=3$, i.e. $Z$ is in linearly general position. Since $\operatorname{deg}(Z) \leq 3 t+1$, the contradiction comes from [8, Theorem 3.2].

Lemma 4. Fix an integer $t>0$. Set $M:=\mathbb{P}^{3}$ and let $Z \subset M$ a zero-dimensional and curvilinear scheme spanning $M$ and with $\operatorname{deg}(Z) \leq 3 t$. We have $h^{1}\left(M, \mathcal{I}_{Z, M}(t)\right)$ $\neq 0$ if and only if either there is a line $R \subset M$ with $\operatorname{deg}(R \cap Z) \geq t+2$ or there is a conic $D \subset M$ such that $\operatorname{deg}(D \cap Z) \geq 2 t+2$.

Proof. The "if " part is trivial. To prove the other implication it is sufficient to exclude the last case of the statement of Lemma 3, By [9, Corollaire 2] (see also 9, Remarque (i)]) we may assume that $h^{1}\left(N, \mathcal{I}_{Z \cap N, N}(t)\right)=0$ for every plane $N$.

Assume that we are in the last case of Lemma 3 and call $L$ the associated line. We may take $Z$ minimal with the property that $h^{1}\left(M, \mathcal{I}_{Z, M}(t)\right)>0$. Let $Q$ be a quadric surface containing $L$ in its singular locus. Since $\operatorname{deg}\left(\operatorname{Res}_{Q}(Z)\right) \leq 3 t-2 t-2 \leq t-2$, we have $h^{1}\left(M, \mathcal{I}_{\operatorname{Res}_{Q}(Z)}(t-2)\right)=0$. Therefore the residual exact sequence of $Q$ gives $h^{1}\left(Q, \mathcal{I}_{Z \cap Q, Q}(t)\right)>0$ and $h^{1}\left(M, \mathcal{I}_{Z \cap Q, M}(t)\right)>0$. The minimality of $Z$ gives $Z \subset Q$. Taking $Q=N_{1}+N_{2}$ in step (b) of the proof of Lemma 3 we also get that only the connected components of $Z$ whose reduction are contained in $L$ arise (for a minimal $Z$ ), hence we reduce to the case $\operatorname{deg}(Z)=2 t+2$.

Let $W \subset Z$ be any degree $2 t+1$ subscheme. Since $\operatorname{deg}(W \cap D) \leq \operatorname{deg}(Z \cap D) \leq t+$ 1 for each line $D$, Lemma 3 gives $h^{1}\left(M, \mathcal{I}_{W, M}(t)\right)=0$. Hence $h^{1}\left(M, \mathcal{I}_{Z, M}(t)\right)=1$. Since $h^{1}\left(N, \mathcal{I}_{Z \cap N, N}(t)\right)=0$ for every plane $N$, as in [7] we get $h^{1}\left(M, \mathcal{I}_{Z, M}(t-1)\right) \geq$ $3+h^{1}\left(M, \mathcal{I}_{Z, M}(t)\right)=4$. Let $N$ be any plane containing $L$. We have $h^{1}\left(N, \mathcal{I}_{Z \cap N}(t-\right.$ $1))=1$, because $\operatorname{deg}(Z \cap L)=t+1$ and $\operatorname{deg}(Z \cap N) \leq 2(t-1)+1$ (use the residual exact sequence of $L$ in $N)$. Since $\operatorname{deg}\left(\operatorname{Res}_{N}(Z)\right) \leq t+1$, we have $h^{1}\left(M, \mathcal{I}_{\operatorname{Res}_{N}(Z)}(t-\right.$ $2)) \leq 2$. Hence the residual exact sequence of $N$ gives $h^{1}\left(M, \mathcal{I}_{\operatorname{Res}_{N}(Z)}(t-1)\right) \leq 2+1$, a contradiction.

Lemma 5. Let $H \subset \mathbb{P}^{4}$ be a hyperplane. Let $S \subset H$ be a set of 12 points in uniform position and spanning $H$.

(a) $h^{1}\left(H, \mathcal{I}_{S, H}(3)\right) \geq 2$ if and only if $S$ is contained in a rational normal curve of $H$ and in this case we have $h^{1}\left(H, \mathcal{I}_{S, H}(3)\right)=2$; 
(b) $h^{1}\left(H, \mathcal{I}_{S, H}(3)\right)=1$ if and only if $S$ is contained in an integral curve $T \subset H$, which is the complete intersection of two quadric surfaces.

Proof. If $S$ is contained in a rational normal curve (resp. an integral complete intersection of two quadric surfaces $)$, then $h^{1}\left(H, \mathcal{I}_{S, H}(3)\right)=2\left(\operatorname{resp} . h^{1}\left(H, \mathcal{I}_{S, H}(3)\right)=\right.$ $1)$. Since $S$ is in linearly general position, we have $h^{1}\left(H, \mathcal{I}_{S^{\prime}}(3)\right)=0$ for each $S^{\prime} \subset S$ with $\sharp\left(S^{\prime}\right)=10$. Hence $h^{1}\left(H, \mathcal{I}_{S, H}(3)\right) \leq 2$. If $h^{0}\left(H, \mathcal{I}_{S, H}(2)\right) \geq 2$, since $S$ is in uniform position we get that $S$ is contained in a integral curve with either degree 3 or the intersection of 2 quadric surfaces. Hence we may assume $h^{0}\left(H, \mathcal{I}_{S, H}(2)\right) \leq 1$. There is $A \subset S$ with $\sharp(A)=8$ and $h^{0}\left(H, \mathcal{I}_{A, H}(2)\right)=2$, i.e. $h^{1}\left(H, \mathcal{I}_{A, H}(2)\right)=0$. Take an ordering $o_{1}, o_{2}, o_{3}, o_{4}$ of $S \backslash A$. Set $A_{0}:=A$. For $i=1,2,3,4$ set $A_{i}:=A \cup\left\{o_{1}, \ldots, i\right\}$. It is sufficient to prove that $h^{0}\left(H, \mathcal{I}_{A_{i}, H}(3)\right)<$ $h^{0}\left(H, \mathcal{I}_{A_{i-1}, H}(3)\right)$ for $i=1,2,3,4$. Let $Q$ be a general quadric surface containing $A$. Since $S$ is in uniform position, we have $Q \cap S=A$. Let $N_{i}$ be any plane not containing $o_{i}$ but containing $o_{j}$ for all $j<i$. The cubic surface $Q \cup A_{i}$ gives $h^{0}\left(H, \mathcal{I}_{A_{i}, H}(3)\right)<h^{0}\left(H, \mathcal{I}_{A_{i-1}, H}(3)\right)$.

Lemma 6. Let $C \subset \mathbb{P}^{r}, r \geq 2$, be a smooth rational curve. Let $M(d, r)$ denote the set of all smooth rational curves of degree $d$ in $\mathbb{P}^{r} . M(d, r)$ is smooth and irreducible of dimension $(r+1) d+r-3$. Set $d:=\operatorname{deg}(C)$ and take a zero-dimensional scheme $Z \subset \mathbb{P}^{n}$ such that $a:=\operatorname{deg}(Z) \leq d+1$. Then $h^{1}\left(N_{C}(-Z)\right)=0$ and the set of all $X \in M(d, r)$ containing $Z$ has dimension $(r+1) d+r-3-(r-1) a$.

Proof. Fix any $Y \in M(d, r)$. Since $T \mathbb{P}^{r}$ is a quotient of $\mathcal{O}_{\mathbb{P}^{r}}(1)$ by the Euler sequence and $X$ is smooth $N_{X}$ is a quotient of $\mathcal{O}_{X}(1)^{(r+1)}$. Since $X$ is a smooth rational curve, we get $h^{1}\left(N_{X}(-W)\right)=0$ for every zero-dimensional scheme $W \subset$ $X$ with $\operatorname{deg}(Z) \leq d+1$. The Hilbert scheme of all curves containing $W$ has $H^{0}\left(N_{X}(-W)\right)$ as its tangent space and $H^{1}\left(N_{X}(-W)\right)$ as an obstruction space ([18, Theorem 1.5]). Taking $W=\emptyset$ we get the smoothness and dimension of $M(d, r)$. The irreducibility of $M(d, r)$ is well-known. Taking $W=Z$ we get the other statements of the lemma.

Let $\mathcal{W}$ denote the set of all quintic hypersurfaces of Cotterill, i.e. satisfying all properties proved in [6]. In particular each $W \in \mathcal{W}$ is a smooth quintic hypersurface containing finitely many rational curves of degree $\leq 11$.

For any integer $b \geq 5$ let $\Delta_{b}$ denote the set of all non-degenerate $C \in M_{12}$ such that there is a line $L \subset \mathbb{P}^{4}$ with $\operatorname{deg}(L \cap C)=b$. Set $\Delta_{7}^{\prime}:=\cup_{b \geq 7} \Delta_{b}$.

Remark 1. For any line $L \subset \mathbb{P}^{4}$ let $A(L, b)$ denote the set of all non-degenerate $C \in M_{12}$ such that $\operatorname{deg}(L \cap C)=b$. Since $\Delta_{b}=\emptyset$ if $b>12$, we have $\operatorname{dim}(A(L, b))=$ $61-2 b$ by Lemma 6. Now, if $W$ is any quintic threefold and $C \subset W$, then by Bezout also $L \subset W$ as soon as $b \geq 6$. Since on each $W \in \mathcal{W}$ there are finitely many lines, if $W$ contains only finitely many $C \in A(L, b)$ for any fixed line $L \subset \mathbb{P}^{4}$, then $W$ contains only finitely many $C \in \Delta_{b}$ as well. Hence to prove that a general $W \in \mathcal{W}$ contains only finitely many elements of $\Delta_{b}$, by (11) and (2) it is sufficient to test the element $C \in \Delta_{b}$ with $h^{1}\left(\mathcal{I}_{C}(5)\right) \geq 2 b+1$.

Lemma 7. A general $W \in \mathcal{W}$ contains only finitely many $C \in \Delta_{7}^{\prime}$.

Proof. By Remark 1 it is sufficient to test the non-degenerate curves $C \in M_{12}$ such that $h^{1}\left(\mathcal{I}_{C}(5)\right) \geq 15$. Take a general hyperplane $H \in \mathbb{P}^{4}$. Since $C \cap H$ is in uniform 
position, Lemma 4 gives $h^{1}\left(H, \mathcal{I}_{C \cap H, H}(t)\right)=0$ for $t=4,5$. The exact sequence

$$
0 \rightarrow \mathcal{I}_{C}(t-1) \rightarrow \mathcal{I}_{C}(t) \rightarrow \mathcal{I}_{C \cap H, H}(t) \rightarrow 0
$$

gives $h^{1}\left(\mathcal{I}_{C}(3)\right) \geq h^{1}\left(\mathcal{I}_{C}(4)\right) \geq h^{1}\left(\mathcal{I}_{C}(5)\right)$. By Lemma 5 we have $h^{1}\left(\mathcal{I}_{C}(2)\right) \geq$ $h^{1}\left(\mathcal{I}_{C}(3)\right)-2 \geq 13$. Hence $h^{0}\left(\mathcal{I}_{C}(2)\right) \geq 3$, contradicting Lemma 1 .

Lemma 8. A general $W \in \mathcal{W}$ contains only finitely many $C \in \Delta_{6}$.

Proof. By Remark 1 it is sufficient to test the non-degenerate curves $C \in M_{12}$ such that $h^{1}\left(\mathcal{I}_{C}(5)\right) \geq 13$. By Lemma 7 we may assume that $C \notin \Delta_{7}^{\prime}$. By Lemmas 2 and 3 we have $h^{1}\left(\mathcal{I}_{C}(4)\right) \geq 4+h^{1}\left(\mathcal{I}_{C}(5)\right) \geq 17$. Take a general hyperplane $H \in \mathbb{P}^{4}$. By Lemma 4 we have $h^{1}\left(H, \mathcal{I}_{C \cap H, H}(4)\right)=0$. The exact sequence (5) gives $h^{1}\left(\mathcal{I}_{C}(3)\right) \geq h^{1}\left(\mathcal{I}_{C}(4)\right)$. By Lemma 5 we have $h^{1}\left(\mathcal{I}_{C}(2)\right) \geq h^{1}\left(\mathcal{I}_{C}(3)\right)-2 \geq 15$. Hence $h^{0}\left(\mathcal{I}_{C}(2)\right) \geq 5$, contradicting Lemma 1

Lemma 9. A general $W \in \mathcal{W}$ contains only finitely many non-degenerate $C \in M_{12}$ such that there is a conic $D \subset \mathbb{P}^{4}$ with $\operatorname{deg}(D \cap C) \geq 10$ and if the conic is singular $C \cap D$ contains a curvilinear scheme of at least degree 10 .

Proof. A conic is either smooth or reducible or a double line. Lemmas 7 and 8 handle the case in which $D$ is not a smooth conic and $\operatorname{deg}(D \cap C) \geq 11$. Assume the existence of a conic $D$ such that $b:=\operatorname{deg}(D \cap C) \geq 10$. Fix any $p \in C \backslash C \cap N$, where $N$ is the plane spanned by $D$, and let $M$ be the hyperplane spanned by $N \cup\{p\}$. Since $\operatorname{deg}(C \cap M) \geq b+1$, we have $b \leq 11$. $\mathbb{P}^{4}$ contains $\infty^{6}$ planes and each plane contains $\infty^{5}$ smooth conics and $\infty^{4}$ singular conic. Fix $b \in\{10,11\}$ and a conic $D$. Let $B(D, b)$ be the set of all non-degenerate $C \in M_{12}$ such that $\operatorname{deg}(D \cap C)=b$; if $b=10$ and $D$ is singular assume that $D \cap C$ is curvilinear. Since each conic contains $\infty^{b}$ curvilinear subschemes of degree $b$, Lemma 6 gives $\operatorname{dim}(B(D, b)) \leq 61-2 b$. Varying $D$ we get that the set of all $C$ has codimension at least 9 in $M_{12}$. Hence it is sufficient to test the curves $C$ with $h^{1}\left(\mathcal{I}_{C}(5)\right) \geq 10$. Since $C \notin \Delta_{7}^{\prime}$, we have $h^{1}\left(\mathcal{I}_{C}(4)\right) \geq 14$. Moreover, if $h^{1}\left(\mathcal{I}_{C}(4)\right)=14$, then $\operatorname{deg}(D \cap C) \geq 10$ for finitely many conics $D_{1}, \ldots, D_{s}$. Let $N_{i}$ be the plane spanned by $D_{i}$. Fix a line $L \subset \mathbb{P}^{4}$ such that $L \cap N_{i}=\emptyset$ for all $i$. Set $V:=H^{0}\left(\mathcal{I}_{L}(1)\right)$ and take any $M \in\left|\mathcal{I}_{L}(1)\right|$. We have $N_{i} \not \subseteq M$. Since $M \cap C$ contains no line $R$ with $\operatorname{deg}(R \cap C) \geq 6$ and no conic $D$ with $\operatorname{deg}(D \cap C) \geq 10$, we have $h^{1}\left(M, \mathcal{I}_{C \cap M, M}(4)\right)=0$ (Lemma 44). Hence the bilinear map $H^{1}\left(\mathcal{I}_{C}(4)\right)^{\vee} \times V \rightarrow H^{1}\left(\mathcal{I}_{C}(3)\right)^{\vee}$ is non-degenerate in the second variable. By the bilinear lemma we have $h^{1}\left(\mathcal{I}_{C}(3)\right) \geq h^{1}\left(\mathcal{I}_{C}(4)\right)+\operatorname{dim}(V)-1=16$. Hence in all cases we have $h^{1}\left(\mathcal{I}_{C}(3)\right) \geq 15$. By Lemma 5 we have $h^{1}\left(\mathcal{I}_{C}(2)\right) \geq 13$, contradicting Lemma 1 .

Let $\Delta_{1}$ (resp. $\Delta_{2}, \Delta_{3}$ ) be the set of all non-degenerate $C \in M_{12}$ such that for a general hyperplane $H \subset \mathbb{P}^{4}$ the set $C \cap H$ is contained in a rational normal curve of $H$ (resp., the smooth complete intersection of 2 quadric surfaces of $H$, resp., a singular integral curve which is the complete intersection of 2 quadric surfaces of $H)$.

We have the following estimates:

Lemma 10. Every irreducible component of $\Delta_{1}$ has dimension $\leq 49$.

Proof. Fix a hyperplane $H$, a rational normal curve $D \subset H$ and $S \subset D$ such that $\sharp(S)=12$. By Lemma 6 the set of all $C \in M_{12}$ containing $S$ has dimension $\leq 61-36$. Since the set of all $S \subset D$ with $\sharp(S)=12$ has dimension 12 and $H$ contains $\infty^{12}$ rational normal curves, we get the lemma. 
Lemma 11. Every irreducible component of $\Delta_{2}$ has dimension $\leq 53$.

Proof. Fix a hyperplane $H$. The set of all degree 4 smooth elliptic curves of $H$ has dimension 16 and we may conclude as in the proof of Lemma 10.

Lemma 12. Every irreducible component of $\Delta_{3}$ has dimension $\leq 52$.

Proof. Fix a hyperplane $H$. The set of all singular, integral and non-degenere curves $D \subset H$ with $\operatorname{deg}(D \cap H)=4$, i.e. the set of all singular integral curves which are the complete intersection of 2 quadric surfaces of $H$, has dimension 15 . Now we argue as in the proof of Lemma 10.

Lemma 13. A general $C \in M_{12}$ contains only finitely many elements of $\Delta_{1} \cup \Delta_{2} \cup$ $\Delta_{3}$.

Proof. By Lemmas 10, 11 and 12, we may assume that $h^{1}\left(\mathcal{I}_{C}(5)\right) \geq 9$. By Lemmas 7. 8 and 9 we may assume $\operatorname{deg}(C \cap L) \leq 5$ for all lines and $\operatorname{deg}(D \cap C) \leq 9$ for all conics. By Lemmas 2 and 3 for $t=4,5$ we get $h^{1}\left(\mathcal{I}_{C}(3)\right) \geq 4+h^{1}\left(\mathcal{I}_{C}(4)\right) \geq 8+$ $h^{1}\left(\mathcal{I}_{C}(5)\right) \geq 17$. Lemma 5 gives $h^{1}\left(\mathcal{I}_{C}(2)\right) \geq 15$, i.e. $h^{0}\left(\mathcal{I}_{C}(2)\right) \geq 5$, contradicting Lemma 1

By Lemmas 7, 8 and 9 to prove Theorem 1 for non-degenerate $C \in M_{12}$ it is sufficient to test the ones such that $\operatorname{deg}(C \cap D) \leq 9$ for any conic $D$ and $\operatorname{deg}(L \cap C) \leq$ 5 for any line $L$. By the cases $t=4,5$ of Lemmas 2 and 3 we have $h^{1}\left(\mathcal{I}_{C}(3)\right) \geq$ $4+h^{1}\left(\mathcal{I}_{C}(4)\right) \geq 8+h^{1}\left(\mathcal{I}_{C}(5)\right)$.

By Lemmas 5 and 13 we may assume $h^{1}\left(H, \mathcal{I}_{C \cap H, H}(3)\right)=0$. Now the case $t=3$ of the exact sequence (5) gives

$$
h^{1}\left(\mathcal{I}_{C}(2)\right) \geq h^{1}\left(\mathcal{I}_{C}(3)\right) \geq 4+h^{1}\left(\mathcal{I}_{C}(4)\right) \geq 8+h^{1}\left(\mathcal{I}_{C}(5)\right) .
$$

Since the stratum in $M_{12}$ corresponding to curves with $h^{1}\left(\mathcal{I}_{C}(5)\right)>0$ has codimension 2 (as in [7. pp. 901-902]), by (11) and (2) we may assume $h^{1}\left(\mathcal{I}_{C}(5)\right) \geq 3$, hence $h^{1}\left(\mathcal{I}_{C}(2)\right) \geq 11$. Since $h^{0}\left(\mathcal{O}_{\mathbb{P}^{4}}(2)\right)=15$ and $h^{0}\left(\mathcal{O}_{C}(2)\right)=25$, we get $h^{0}\left(\mathcal{I}_{C}(2)\right) \geq 1$.

Now, if $h^{1}\left(\mathcal{I}_{C}(5)\right) \leq 5$ (hence $h^{0}\left(\mathcal{I}_{C}(5)\right) \leq 70$ ) we conclude by the following Lemma 14.

Lemma 14. Let $\Gamma$ be any irreducible family of non-degenerate curves of $M_{d}, d>1$, contained in some quadric hypersurface. Then $\operatorname{dim} \Gamma \leq 14+3 d$.

Proof. Since $\operatorname{dim}\left|\mathcal{O}_{\mathbb{P}^{4}}(2)\right|=14$ and singular quadrics occur in codimension 1 , it is sufficient to prove that for every smooth (resp., integral but singular) quadric $Q$ the set $\Gamma^{\prime}$ of all $C \in M_{d}$ contained in $Q$ has dimension $\leq 3 d$ (resp., $\leq 3 d+1$ ).

First assume that either $Q$ is smooth or $C$ does not intersect the singular locus $V$ of $Q$. In this case the normal sheaf $N_{C, Q}$ is a rank 2 spanned vector bundle on $C$, hence $h^{1}\left(N_{C, Q}\right)=0$. Since $\operatorname{det}\left(N_{C, Q}\right)$ has degree $3 d-2$ and $N_{C}$ has rank 2, Riemann-Roch gives $h^{0}\left(N_{C, Q}\right)=3 d$, proving the lemma in this case.

Now assume $C \cap V \neq \emptyset$ and set $x:=\operatorname{deg}(C \cap V)$. Since $C$ is smooth, $x=1$ if $\operatorname{dim}(V)=0$. Let $\tau_{Q}$ denote the tangent sheaf of $Q$. The vector space $H^{0}\left(\tau_{Q}\right)$ is the tangent space at the identity map of the automorphism group $\operatorname{Aut}(Q)$. Since $Q \backslash V$ is homogeneous, $\tau_{Q} \mid(Q \backslash V)$ is a spanned vector bundle. Since $C$ is not a line and $\operatorname{dim} V \leq 1$, the set $V \cap C$ is finite. Dualizing the natural map from the conormal sheaf of $C$ in $Q$ to $\Omega_{Q}^{1}$ we get a map $u: \tau_{Q} \mid C \rightarrow N_{C, Q}$ which is surjective outside the finite set $C \backslash C \cap V$. Since $C$ is smooth and rational and $\tau_{Q}$ is spanned at each 
point of $Q \backslash V$, we get $h^{1}\left(N_{C, Q}\right)=0$. Since we need to prove that $\operatorname{dim} \Gamma^{\prime} \leq 3 d+1$, it is sufficient to check this inequality when $C$ is a general element of $\Gamma^{\prime}$. In particular we may assume that $\operatorname{deg}\left(C^{\prime} \cap V\right)=x$ for a general $C^{\prime} \in \Gamma^{\prime}$ and use induction on the integer $x$, the case $x=0$ being true by the case $C \cap V=\emptyset$ proved before. Set $\Gamma^{\prime \prime}:=\left\{C^{\prime} \in \Gamma^{\prime}: \operatorname{deg}(V \cap C)=x\right\}$. It is sufficient to prove that $\operatorname{dim} \Gamma^{\prime \prime} \leq 3 d+1$. Let $v: \widetilde{Q} \rightarrow Q$ be the blowing up of $V, E:=v^{-1}(V)$ the exceptional divisor, and $\widetilde{C} \subset \widetilde{Q}$ the strict transform of $C$. Since $C$ is smooth, $v$ maps isomorphically $\widetilde{C}$ onto $C$ and the numerical class of $\widetilde{C}$ with respect to $\operatorname{Pic}(\widetilde{Q})$ only depends on $\operatorname{dim}(V), d$ and $x$. Let $\Psi$ be closure in $\operatorname{Hilb}(\widetilde{Q})$ of the strict transforms of all $C^{\prime} \in \Gamma^{\prime \prime}$. It is sufficient to prove that $\operatorname{dim} \Psi \leq 3 d+1$. Take a general $D \in \Psi$. Since $\operatorname{Aut}(\widetilde{Q})$ acts transitively of $\widetilde{Q} \backslash E$, the first part of the proof gives $h^{1}\left(N_{D, \widetilde{Q}}\right)=0$. Hence it is sufficient to prove that $\operatorname{deg}\left(N_{D, \widetilde{Q}}\right) \leq 3 d-1$, i.e. $\operatorname{deg}\left(\tau_{\widetilde{Q}}\right) \leq 3 d+1$, i.e. $\operatorname{deg}\left(\omega_{\widetilde{Q}} \mid D\right) \geq-3 d-1$. The group $\operatorname{Pic}(\widetilde{Q})$ is freely generated by $E$ and the pull-back $H$ of $\mathcal{O}_{Q}(1)$. We have $D \cdot H=d$ and $D \cdot E=x$. We have $\omega_{\widetilde{Q}} \cong \mathcal{O}_{\widetilde{Q}}(-3 H+c E)$ with $c=-1$ if $\operatorname{dim}(V)=0$ (see for instance [12, Example $8.5(2))$ and $c=0$ if $\operatorname{dim}(V)=1$ (see for instance 12. Example $8.5(3))$. Hence $\operatorname{deg}\left(\omega_{\widetilde{Q}_{\mid D}}\right)=-3 d+c x \geq-3 d-1$ and the proof is complete.

If instead $h^{1}\left(\mathcal{I}_{C}(5)\right) \geq 6$, then by (6) we have $h^{1}\left(\mathcal{I}_{C}(2)\right) \geq 14$, i.e. $h^{0}\left(\mathcal{I}_{C}(2)\right) \geq 4$, contradicting Lemma 1 .

\section{Degenerate CASE}

The degenerate case occurs in codimension 10 of $M_{12}$. Indeed, the general curve of degree $d=12$ in $\mathbb{P}^{3}$ has maximal rank (1]), in particular it does not sit on any quintic. It follows that our codimension is $\operatorname{dim}\left(M_{d}\right)-(4 d-1+4)=61-51=10$. Hence we may assume $h^{1}\left(\mathcal{I}_{C}(5)\right) \geq 11$.

We consider degenerate curves $C \in M_{12}$ with $h^{1}\left(\mathcal{I}_{C}(5)\right) \geq 11$ contained in a hyperplane $M$ and in a general quintic $W$ with $W^{\prime}:=M \cap W$.

Lemma 15. Let $W \subset \mathbb{P}^{4}$ be a general quintic hypersurface. Then $W$ contains finitely many integral curves $T$ of degree 4 which are the complete intersection of a hyperplane and 2 quadric hypersurfaces and all of them are smooth.

Proof. Since $W$ contains no singular rational curves ([6]), it is sufficient to consider the smooth ones, i.e. the degree 4 elliptic curves of $\mathbb{P}^{4}$. Let $\Gamma^{\prime}$ be the set of all degree 4 elliptic curves of $\mathbb{P}^{4}$. Fix $T \in \Gamma^{\prime}$. Since $N_{T} \cong \mathcal{O}_{T}(2)^{\oplus 2} \oplus \mathcal{O}_{T}(1)$, we have $h^{1}\left(N_{T}\right)=0$, hence $\Gamma^{\prime}$ is smooth and of dimension $\chi\left(N_{T}\right)=16$. Since $T$ is a complete intersection, we have $h^{1}\left(\mathcal{I}_{T}(5)\right)=0$ and $h^{0}\left(\mathcal{I}_{T}(5)\right)=\left(\begin{array}{l}9 \\ 4\end{array}\right)-20$. Hence a dimension count gives the lemma.

Lemma 16. $W^{\prime}$ contains only finitely many non-degenerate curves of degree 5 and 6.

Proof. Fix a degree $t \in\{5,6\}$ integral and non-degenerate curve $D \subset W^{\prime}$ and set $q:=p_{a}(D)$. By [10] we have $h^{1}\left(M, \mathcal{I}_{D, M}(5)\right)=0$, hence $h^{0}\left(\mathbb{P}^{4}, \mathcal{I}_{D}(5)\right)=$ $126-5 t-1+q$.

First assume $t=5$. By the genus bound for space curves we have $q \leq 2$. Since $q \leq 2$, we have $h^{1}\left(\mathcal{O}_{D}(1)\right)=0$ and in particular $h^{1}\left(\mathcal{O}_{D}(5)\right)=0$, i.e. $h^{0}\left(\mathcal{O}_{D}(5)\right)=$ $5 t+1-q$. Since $q \leq 2$, all the irreducible components of the Hilbert scheme of $M$ 
containing $D$ have dimension 20 . Since $\mathbb{P}^{4}$ has $\infty^{4}$ hyperplanes, it is sufficient to use that $4 t+4 \leq 5 t+1-q$.

Now assume $t=6$. By the genus bound for space curves ([11, Theorems 3.7 and 3.13]), $q \leq 3$ and $q=3$ if and only if $D$ is contained in a quadric surface $Q$. Assume $q=3$. In this case $D$ is the complete intersection of $Q$ and a cubic surface ([11, Corollary 3.14]) and so $D$ is a locally complete intersection, $\omega_{D} \cong \mathcal{O}_{D}(1)$ and $N_{D, M} \cong \mathcal{O}_{D}(2) \oplus \mathcal{O}_{D}(3)$. Since $h^{1}\left(N_{D}\right)=0$ the Hilbert scheme of $M$ at $D$ is smooth and of dimension $4 t$. We conclude as in the case $t=5$. The case $q \leq 2$ is done as in the case $t=5$.

A theorem of Zak (see for instance 22] ) states that the Gauss map of any smooth projective variety is finite, hence $W^{\prime}$ has only finitely many singular points, all of them being hypersurface singularities. By [15, p. 733] $W^{\prime}$ has only rational double points of type $A_{i}, i \leq 4$, and $D_{4}$ as singularities.

We may improve the lower bound $h^{1}\left(\mathcal{I}_{C}(5)\right) \geq 11$ if we restrict the set of hyperplanes or rather if we restrict the pairs $(W, M) \in\left|\mathcal{O}_{\mathbb{P}^{4}}(5)\right| \times\left|\mathcal{O}_{\mathbb{P}^{4}}(1)\right|$.

Remark 2. If $M$ is tangent to $W$, i.e. if $W^{\prime}$ is singular, then we may assume $h^{1}\left(\mathcal{I}_{C}(5)\right) \geq 12$. Since the Gauss map is birational, if $W^{\prime}$ has at least two singular points, then we may assume $h^{1}\left(\mathcal{I}_{C}(5)\right) \geq 13$.

Remark 3. For any line $L \subset \mathbb{P}^{4}$ we have $h^{0}\left(\mathcal{I}_{L}(1)\right)=3$. A general $W$ contains only finitely many lines $\left([6)\right.$. Hence if $W^{\prime}$ contains a line, then we may assume $h^{1}\left(\mathcal{I}_{C}(5)\right) \geq 13$. Since any two lines of $W$ are disjoint ([6]), any two lines of $W$ span a hyperplane. Hence if $W^{\prime}$ contains two different lines, then we may assume $h^{1}\left(\mathcal{I}_{C}(5)\right) \geq 15$. Fix a line $L \subset W$. For any $p \in L$, the hyperplane $T_{p} W$ is the only hyperplane singular at $p$. Since $\operatorname{dim}(L)=1$, we get that if $W^{\prime}$ is singular at one point of $L$, then we may assume $h^{1}\left(\mathcal{I}_{C}(5)\right) \geq 14$.

Remark 4. For any smooth conic $D \subset \mathbb{P}^{4}$ we have $h^{0}\left(\mathcal{I}_{D}(1)\right)=2$. A general $W$ contains only finitely many conics ([6]). Hence if $W^{\prime}$ contains a smooth conic, then we may assume $h^{1}\left(\mathcal{I}_{C}(5)\right) \geq 14$.

Remark 5. For any integer $x$ with $3 \leq x \leq 11, W$ contains only finitely many smooth rational curve of degree $x$, none of them contained in a plane. Hence if $W^{\prime}$ contains a smooth rational curve of degree $x$, then we may assume $h^{1}\left(\mathcal{I}_{C}(5)\right) \geq 15$. The same is true if $W^{\prime}$ contains a line and a conic or 2 conics.

For any hyperplane $U$ let $M_{12}(U)$ denote the set of all $C \in M_{12}$ contained in $U$. The locus $M_{12}(U)$ is smooth and irreducible and $\operatorname{dim}\left(M_{12}(U)\right)=48$.

Remark 6. Fix an integer $e>0$ and assume the existence of a line $L \subset W^{\prime}$ such that $\operatorname{deg}(L \cap C)=e$. Let $\mathcal{J}(e)$ be the set of all quadruples $(W, H, L, C)$ with $W \in \mathcal{W}$, $H$ a hyperplane, $L \subset W \cap U$ a line, $C \in M_{12}(U)$ and $\operatorname{deg}(L \cap C)=e$. Fix any $(W, H, L, C) \in \mathcal{J}(e)$. We have $\mathcal{J}(e)=\emptyset$ if $e \geq 12$. Now assume $e \leq 11$. Fix a line $L \subset M$ and a degree $e$ zero-dimensional scheme $Z \subset U$ with $\operatorname{deg}(Z)=e$ and take any $C \in M_{12}(U)$ such that $Z \subset C$. As in Lemma 6 we see that $h^{1}\left(N_{C, M}(-Z)\right)=0$, hence the set of all $C \in M_{12}(U)$ with $Z \subset C$ has dimension $48-2 e$. Varying $Z$ in $L$ we see that the set of all $C \in M_{12}(U)$ the set of all $C \in M_{12}(U)$ such that $\operatorname{deg}(C \cap L)=e$ has dimension $\leq 48-e$. Since each $W \in \mathcal{W}$ contains only finitely many lines, to show that for all $(W, M, L, C) \in \mathcal{J}(e)$ we have $C \nsubseteq W$ it is sufficient to exclude the ones with $h^{1}\left(\mathcal{I}_{C}(5)\right) \geq 13+e$. 
Since the Gauss map of the smooth projective variety $W$ is finite, $W^{\prime}$ has only finitely many singular points. Since $W^{\prime}$ is locally a complete intersection, $W^{\prime}$ is normal. By [6] $W$ has only finitely many lines and only finitely many conics and no singular rational curve of degree $\leq 11$. By Lemma $15 W$ has only finitely many smooth elliptic curves of degree 4.

Remark 7. Let $W \subset \mathbb{P}^{4}$ be a general quintic. Let $\mathcal{D}_{i}, i \geq 1$, be the set of all irreducible plane curves of degree $i$ contained in $W$. Since $W$ contains no plane, we have $\mathcal{D}_{i}=\emptyset$ for all $i \geq 6$, and the set $\mathcal{D}_{5}$ is formed by the irreducible degree 5 curves of the form $W \cap N$ with $N \subset \mathbb{P}^{4}$ a plane. Hence $\mathcal{D}_{5}$ is irreducible and of dimension 8. By 6, $\mathcal{D}_{1} \cup \mathcal{D}_{2}$ is finite and any two elements of it are disjoint. Fix $D \in \mathcal{D}_{3}$ and let $N \subset \mathbb{P}^{4}$ be the plane spanned by $D$. The plane curve $W \cap N$ is the union of some $D \in \mathcal{D}_{3}$ together with a smooth conic, a reducible conic, or a double line. Since $\mathcal{D}_{2}$ is finite, the first case may occur only for finitely many planes and these are exactly the planes $N$ such that $W \cap N=T_{2} \cup T_{3}$ with $T_{2} \in \mathcal{D}_{2}$ and $T_{3} \in \mathcal{D}_{3}$. The second case does not occur, because the lines of $W$ are disjoint. Now assume that $W \cap N=D \cup 2 L$ with $L$ a line. By Zak's tangency theorem the restriction to $L$ of the Gauss map of $W$ is finite. Therefore the third case occurs only for at most one plane $N \supset L$ Now take $T \in \mathcal{D}_{4}$ and let $N$ be the plane spanned by $T$. We have $N \cap W=T \cup R$ with $R \in \mathcal{D}_{1}$, hence all elements of $\mathcal{D}_{4}$ are obtained in the following way. Since the set of all planes of $\mathbb{P}^{4}$ containing a line is a 2 -dimensional projective space, each irreducible component of $\mathcal{D}_{4}$ has dimension 2. Fix any $L \in \mathcal{D}_{1}$ and take the intersection with $W$ of the element of the net of all planes of $\mathbb{P}^{4}$ containing $L$. For a fixed hyperplane $M \subset L$ the set of all planes containing $L$ and contained in $M$ has dimension 1.

Let $\alpha$ be the minimal degree of a surface of $M$ containing $C$. Since $C$ is irreducible, every degree $\alpha$ surface containing $C$ is irreducible.

Lemma 17. We have $\alpha>3$.

Proof. Since $C$ spans $M$, we have $\alpha>1$. Assume $\alpha=2$ and take $Q \in\left|\mathcal{I}_{C, M}(2)\right|$. Since $W^{\prime}$ is irreducible, $W^{\prime} \cap Q$ is a degree 10 curve containing $C$, a contradiction. Now assume $\alpha=3$. Since $\operatorname{deg}(C)>9, C$ is contained in a unique cubic surface $S$. Let $J \subset S \cap W^{\prime}$ be the locally Cohen-Macaulay curve linked to $C$ by the complete intersection $S \cap W^{\prime}$. We have $\operatorname{deg}(J)=3$ and $p_{a}(J)=-18$ (19, Proposition 3.1]). Since $\operatorname{deg}(J)<-p_{a}(J), J$ has a multiple component. Since $\operatorname{deg}(J)=3$, the multiple component is a line, $L$. Since $\left|\mathcal{I}_{C, M}(5)\right|$ contains all quintic surfaces $S \cup Q$ with $Q \in\left|\mathcal{O}_{M}(2)\right|$ and $W^{\prime}$ is irreducible, we have $h^{0}\left(M, \mathcal{I}_{C, M}(5)\right) \geq 11$, i.e. $h^{1}\left(M, \mathcal{I}_{C, M}(5)\right) \geq 16$. Assume for the moment the non-existence of a line $R \subset M$ with $\operatorname{deg}(R \cap C) \geq 7$. By Lemmas 2 and 3 we get $h^{1}\left(M, \mathcal{I}_{C, M}(4)\right) \geq 19$. Fix a general plane $N \subset M$. We have an exact sequence

$$
0 \rightarrow \mathcal{I}_{C, M}(t-1) \rightarrow \mathcal{I}_{C, M}(t) \rightarrow \mathcal{I}_{C \cap N, N}(t) \rightarrow 0
$$

Since $N$ is general, the plane cubic $C \cap N$ is irreducible and $C \cap N$ is formed by 12 points of the smooth locus of $C \cap N$. Hence $h^{1}\left(N, \mathcal{I}_{C \cap N, N}(4)\right) \leq 1$ with equality if and only if $C \cap N$ is the complete intersection of $S \cap N$ with a plane quartic. Since $h^{0}\left(M, \mathcal{I}_{C, M}(2)\right)=0$ and (by the genus formula) $C$ is not a complete intersection of two surfaces, [21, Theorem 6] gives $h^{1}\left(N, \mathcal{I}_{C \cap N, N}(4)\right)=0$. The case $t=4$ of (77) gives $h^{1}\left(M, \mathcal{I}_{C, M}(3)\right) \geq 19$, i.e. $h^{0}\left(M, \mathcal{I}_{C, M}(3)\right) \geq 2$, a contradiction. Now assume the existence of a line $R \subset M$ such that $e:=\operatorname{deg}(R \cap C) \geq 7$. 
There are at most finitely many such $R$, because they cannot be all the lines of a ruling of $S$. Take a line $L \subset M$ disjoint from all $R$. Set $V:=H^{0}\left(M, \mathcal{I}_{L, M}(1)\right)$. Take any plane $U \subset M$ containing $L$. Since $\operatorname{deg}(K \cap C) \leq 6$ for each line $K \subset$ $U$, we have $h^{1}\left(U, \mathcal{I}_{C \cap U, U}(5)\right)=0$. Hence the bilinear map $H^{1}\left(M, \mathcal{I}_{C, M}(5)\right)^{\vee} \times$ $V \rightarrow H^{1}\left(\mathcal{I}_{C, M}(4)\right)^{\vee}$ is non-degenerate. Since $\operatorname{dim}(V)=2$, the bilinear lemma gives $h^{1}\left(M, \mathcal{I}_{C, M}(4)\right) \geq h^{1}\left(M, \mathcal{I}_{C, M}(5)\right)+2-1$. Since $e>5$, Bezout gives $R \subset$ $W^{\prime}$. By Remark 6 we may assume $h^{1}\left(M, \mathcal{I}_{C, M}(5)\right) \geq 20$. Since we just proved that $h^{1}\left(N, \mathcal{I}_{C \cap N, N}(4)\right)=0$, we get $h^{1}\left(M, \mathcal{I}_{C, M}(3)\right) \geq 21$, hence the contradiction $h^{0}\left(M, \mathcal{I}_{C, M}(3)\right) \geq 3$.

Lemma 18. $W^{\prime}$ contains no $C \in M_{12}(M)$ such that $h^{0}\left(M, \mathcal{I}_{C, M}(4)\right) \geq 3$ and no $C \in M_{12}(M)$ with a line $L \subset M$ with $\operatorname{deg}(L \cap C) \geq 7$.

Proof. The statement is made of two parts.

(a) Take general $S_{1}, S_{2} \in\left|\mathcal{I}_{C, M}(4)\right|$ and take a general $\left(S_{1}, S_{2}\right) \in\left|\mathcal{I}_{C, M}(4)\right|^{2}$. Since $\alpha>3, S_{i}$ is irreducible. The complete intersection $S_{1} \cap S_{2}$ links $C$ to a degree 4 curve $J$ with $p_{a}(J)=-16$ ([19, Proposition 3.1]), hence $J$ has at least one multiple component, say $B$ with multiplicity $c \geq 2$. Since $h^{0}\left(M, \mathcal{I}_{C, M}(4)\right) \geq 3, J$ has also a movable component $A$. Hence $B$ is a line and either $A$ is a line or it is a smooth conic.

First assume that $A$ is a smooth conic, $c=2$, and $J$ has no other component. We have $p_{a}(C \cup B)=\operatorname{deg}(C \cap B)-1 \leq 10$ and $p_{a}(A \cup B)=\operatorname{deg}(A \cap B)-1 \geq-1$. Since $A \cup B$ is linked to $C \cup A$ by the complete intersection $S_{1} \cap S_{2}$, we have $p_{a}(A \cup B)=p_{a}(C \cup B)-20 \leq-10$, a contradiction.

Now assume $\operatorname{deg}(A)=1$. Moving $S_{2}$ we get that $S_{1}$ is ruled by lines. Since $\operatorname{deg}\left(S_{1}\right)>2, S_{1}$ has a unique ruling. This case cannot occur if $h^{0}\left(M, \mathcal{I}_{C, M}(4)\right) \geq 4$, because the plane is the only surface with $\infty^{2}$ lines.

First assume that $c=2$. In this case $J$ contains a line $R \notin\{B, A\}$. We have $p_{a}(C \cup B) \leq 10, p_{a}(B \cup A \cup R) \geq-2$, while [19, Proposition 3.1] gives $p_{a}(B \cup A \cup R)=p_{a}(C \cup B)-20$, a contradiction. Now assume $c=3$. $C, A$ and $B$ are the unique components of $S_{1} \cap S_{2} . S_{i}$ and $S_{2}$ do not contain $B$ in their singular locus, because $S_{1} \cap S_{2}$ would contain $B$ with multiplicity 2 . Since the line $B$ is not a line of the ruling of $S_{1}, S_{1}$ is not a cone, it is rational and it is a linear projection from a minimal degree surface $S \subset \mathbb{P}^{5}$ (neither the Veronese surface not a cone). $S$ is a Hirzebruch surface, either $F_{0} \cong \mathbb{P}^{1} \times \mathbb{P}^{1}$ embedded by the complete linear system $\left|\mathcal{O}_{F_{0}}(h+2 f)\right|$ or $F_{2}$ embedded by the complete linear system $|h+3 f| . S_{1}$ is not a linear projection of $F_{0}$, because it has a line, $B$, not in the ruling and not in the singular locus (i.e. the image of a conic of $F_{0}$ ). Hence $S_{1}$ is a linear projection of $F_{0}$. Any smooth rational curve $C_{1} \subset F_{0}$ with $C_{1}$ not a line is an element of $|h+x f|$ for some $x \geq 3$. We have $\operatorname{deg}\left(C_{1}\right)=(h+x f) \cdot(h+3 f)=x$, hence if $C_{1}$ has $C$ as its projection, then $x=12$. $B$ is the image of $h$. We have $\operatorname{deg}\left(h \cap C_{1}\right)=10$ and so $\operatorname{deg}(C \cap B) \geq 7$. Hence to prove the lemma it is sufficient to prove the second assertion.

(b) Take $C \in M_{12}(M)$ with a line $L \subset M$ with $\operatorname{deg}(L \cap C) \geq 7$. By part (a) to get a contradiction it is sufficient to prove that $h^{0}\left(M, \mathcal{I}_{C, M}(4)\right) \geq 4$. Bezout gives $R \subset W^{\prime}$. By Remark 6 we may assume $h^{1}\left(M, \mathcal{I}_{C, M}(5)\right) \geq 19$. As in the proof of Lemma 17 we get $h^{1}\left(M, \mathcal{I}_{C, M}(4)\right) \geq 20$, i.e. $h^{0}\left(M, \mathcal{I}_{C, M}(4)\right) \geq 6$.

Remark 8. Take $C \in M_{12}(M)$ without lines $L$ with $\operatorname{deg}(L \cap C) \geq 7$. By Lemma 2 to prove that $h^{0}\left(M, \mathcal{I}_{C, M}(4)\right) \geq 3$ (hence to prove that $C \nsubseteq W^{\prime}$ by Lemma 18) 
it is sufficient to prove that $h^{1}\left(\mathcal{I}_{C}(5)\right) \geq 14$. By Remarks 3 , 4 and 5 this is always the case if $W^{\prime}$ contains a smooth rational curve of degree $\geq 2$ or if it contains two lines. So from now on we assume that $W^{\prime}$ has no such curves, hence no smooth elliptic curve of degree 3 by Remark 7 We also assume that $W^{\prime}$ has no smooth elliptic curve of degree 4 by Lemma 15 .

Now we are going to apply all of the dimension-counting remarks and lemmas above and to use liaison in order to show that degenerate rational curves which are sufficiently generic (with respect to the properties described in the remarks and lemmas) must in fact have $h^{1}\left(\mathcal{I}_{C}(5)\right)<11$, contradiction. Our argument hinges on a careful case-by-case analysis involving the types of divisors that that arise as components of certain residuals $C_{T}$ to $C$ inside of complete intersections of type $(5,5)$.

Since $h^{1}\left(\mathcal{I}_{C}(5)\right) \geq 11$ we have $h^{0}\left(M, \mathcal{I}_{C}(5)\right) \geq 6$. Hence $h^{0}\left(W^{\prime}, \mathcal{I}_{C, W^{\prime}}(5)\right) \geq 5$. For any $\left.T \in \mid \mathcal{I}_{C, M}(5)\right) \mid$ with $T \neq W^{\prime}$ let $C_{T} \subset T \cap W^{\prime}$ denote the curve linked to $C$ by the complete intersection $T \cap W^{\prime}$.

We have $\operatorname{deg}\left(C_{T}\right)=13, \chi\left(\mathcal{O}_{C_{T}}\right)=-2\left(\left[19\right.\right.$, Proposition 3.1]) and $h^{1}\left(\mathcal{I}_{C_{T}}(1)\right)=$ $h^{1}\left(\mathcal{I}_{C}(5)\right)$ ([16, Theorem 1.1 (a)], [20]). Since $\operatorname{deg}\left(C_{T}\right)=13, \chi\left(\mathcal{O}_{C_{T}}\right)=-2, C_{T}$ is not a plane curve (i.e. $\left.h^{0}\left(M, \mathcal{I}_{C_{T}}(1)\right)=0\right)$, hence $h^{0}\left(\mathcal{O}_{C_{T}}(1)\right)=h^{1}\left(\mathcal{I}_{C_{T}}(1)\right)+4=$ $h^{1}\left(\mathcal{I}_{C}(5)\right)+4 \geq 15$. Since $\operatorname{deg}\left(C_{T}\right)>2 p_{a}\left(C_{T}\right)-2$, we see that $C_{T}$ is not integral.

Varying $T$ we find inside $W^{\prime}$ a positive dimensional family of effective divisors $C_{T}$, all of them linked to $C$ and with the same arithmetic genus, hence a flat family of effective divisors of $W^{\prime}$. Therefore some of the effective divisors whose sum gives $C_{T}$ moves in $W^{\prime}$.

Let $D_{1}, \ldots, D_{k}$ be all all movable divisors of $C_{T}$ and let $R_{1}, \ldots, R_{u}$ the fixed divisors with multiplicities $e_{1}, \ldots, e_{u}$. Hence for a general $T$ we have $C_{T}=D_{1}+$ $\cdots+d_{k}+e_{1} R_{1}+\cdots e_{u} R_{u}$ as effective Weil divisors of $W^{\prime}$.

Let $m\left(D_{i}\right), 1 \leq i \leq k$, be the dimension of $D_{i}$ in the family $\left.\mid \mathcal{I}_{C, W^{\prime}}(5)\right) \mid$. We have $m\left(D_{1}\right)+\cdots+m\left(D_{k}\right) \geq 4$. We also proved that $m\left(D_{1}\right)+\cdots+m\left(D_{k}\right) \geq h^{1}\left(\mathcal{I}_{C}(5)\right)-7$. We saw that if $\operatorname{deg}\left(D_{i}\right)=4$, then $W^{\prime}$ contains a line $L$ and $m\left(D_{i}\right)=1$, because the moving family is induced by the family of planes of $M$ containing $L$. We saw that if $\operatorname{deg}\left(D_{i}\right)=5$, then $D_{i}$ is a plane section of $W^{\prime}$, hence $m\left(D_{i}\right)=3$.

Let $R_{1}, \ldots, R_{u}, u \geq 0$, be the fixed divisors of $\left|\mathcal{I}_{W^{\prime}, C}(5)\right|$ and call $b_{i}$ the multiplicity of $R_{i}$ in $C_{T}$ (for a general $T$ ).

(a) Assume the existence of $i \in\{1, \ldots, k\}$ such that $D_{i}$ is a plane curve of degree 5 . With no loss of generality we may assume $i=1$. Let $N$ be the plane containing $D_{1}$. Since $m\left(D_{1}\right)=3$, we have $k \geq 2$ and $h^{0}\left(W^{\prime}, \mathcal{I}_{C \cup D_{1}, W^{\prime}}(5)\right) \geq$ 2. Since $h^{0}\left(W^{\prime}, \mathcal{I}_{C \cup D_{1}, W^{\prime}}(5)\right)=h^{0}\left(W^{\prime}, \mathcal{I}_{C, W^{\prime}}(4)\right)$. Since $h^{1}\left(M, \mathcal{I}_{W^{\prime}, M}(4)\right)=$ $h^{1}\left(\mathcal{O}_{M}(-1)\right)=0$, we get $h^{0}\left(M, \mathcal{I}_{C}(4)\right) \geq 2$. Since $\alpha>3$ (Lemma 17), there are integral quartic surfaces $T_{i} \in\left|\mathcal{I}_{C, M}(4)\right|, i=1,2$, with $T_{1} \neq T_{2}$. The complete intersection $T_{1} \cap T_{2}$ links $C$ to a locally Cohen-Macaulay curve $G$ such that $\operatorname{deg}(G)=4$. and $p_{a}(G)=-16$ ([19, Proposition 3.1]). Since $p_{a}(G) \leq-4$ and $\operatorname{deg}(G)=4, G$ has at least one multiple component, $J$, with multiplicity $e \geq 2$. Our proof of the existence of $T_{1}$ and $T_{2}$ shows that we may take $T_{1}, T_{2}$ such that $D_{2}$ is a subcurve of $G$. Since $\operatorname{deg}\left(D_{2}\right) \geq 4=\operatorname{deg}(G)$ and $G$ has a multiple component, we get a contradiction.

(b) Assume the existence of $i \in\{1, \ldots, k\}$ such that $D_{i}$ is a plane curve of degree 4. Just to fix the notation we assume $i=1$. Let $N$ be the plane spanned by $D_{1}$. We have $W^{\prime} \cap N=D_{1} \cup L$ with $L$ a line. Remark 3 gives 
$h^{0}\left(\mathcal{I}_{C}(5)\right) \geq 7$, hence $m\left(D_{1}\right)+\cdots+m\left(D_{k}\right) \geq 6$. We saw that $m\left(D_{1}\right)=1$. Since $m\left(D_{2}\right)+\cdots+m\left(D_{k}\right) \geq 5$, we have $h^{0}\left(W^{\prime}, \mathcal{I}_{C \cup D_{1}, W^{\prime}}(5)\right) \geq 6$. Since $\operatorname{deg}\left(L \cap D_{1}\right)=$ 4 , we get $h^{0}\left(W^{\prime}, \mathcal{I}_{C \cup D_{1} \cup L}(5)\right) \geq 4$. Hence we may find a movable divisor $E$ in $\left|\mathcal{I}_{C \cup L \cup D_{1}}(5)\right|$. We saw that $\operatorname{deg}(E) \geq 4$. As in step (a) we get $h^{0}\left(M, \mathcal{I}_{C, M}(4)\right) \geq 4$, contradicting Lemma 18

(c) From now on we assume that each $D_{i}$ is non-degenerate. By Lemma 16 we may assume $\operatorname{deg}\left(D_{i}\right) \geq 7$ for all $i$. By Remark 8 we cannot have $2 \leq \operatorname{deg}\left(R_{i}\right) \leq 4$ and we have $\operatorname{deg}\left(R_{i}\right)=1$ at most one index $i$.

Recall that $13=\sum_{i=1}^{k} \operatorname{deg}\left(D_{i}\right)+\sum_{i=1}^{u} b_{i} R_{u}$ and we proved that $k+u>1$. Since $\operatorname{deg}\left(D_{i}\right) \geq 7$ for all $i$, we have $k=1$.

Assume that $C_{T}$ has no multiple component. We have $h^{0}\left(\mathcal{O}_{D_{1}}(1)\right)+h^{0}\left(\mathcal{O}_{R_{1}}(1)\right)+$ $\cdots+h^{0}\left(\mathcal{O}_{R_{u}}(1)\right) \geq 2+h^{1}\left(\mathcal{I}_{C}(5)\right)$. Since $D_{1}$ moves, we have $p_{a}\left(D_{1}\right)>0([\underline{6})$, hence $h^{0}\left(\mathcal{O}_{D_{1}}(1)\right) \leq \operatorname{deg}\left(D_{1}\right)$. Since $h^{0}\left(\mathcal{O}_{R_{i}}(1)\right)=\operatorname{deg}\left(R_{i}\right)+1$ for at most one index $i$, we get a contradiction.

Hence $C_{T}$ has at least one multiple component, say $R_{1}$. Since $\operatorname{deg}\left(D_{1}\right) \geq 6$, we get $b_{1} \operatorname{deg}\left(R_{1}\right) \leq 13-\operatorname{deg}\left(D_{1}\right) \leq 7$ and in particular $\operatorname{deg}\left(R_{1}\right) \leq 3$. Since $W^{\prime}$ has no curve of degree 2 or $3(\operatorname{Remark} 8), R_{1}$ is a line, hence we may assume $h^{1}\left(\mathcal{I}_{C}(5)\right) \geq$ 13. Set $b:=b_{1}, R:=R_{1}$ and $e:=\operatorname{deg}(C \cap R)$. We have $\operatorname{deg}\left(D_{1}\right)=13-b$. By Remarks 6 and 8 we may assume $e=0$, i.e. $R \cap C=\emptyset$ and that $R$ is contained in the smooth locus of $W^{\prime}$.

(d) Recall that $h^{1}\left(M, \mathcal{I}_{C, M}(5)\right) \geq 13$. By Lemma 18 we may assume that $L$ has no line $T$ with $\operatorname{deg}(T \cap C) \geq 7$. By Lemma 2 we have $h^{1}\left(M, \mathcal{I}_{C, M}(4)\right) \geq 16$, i.e. $h^{0}\left(M, \mathcal{I}_{C, M}(4)\right) \geq 2$. Since $\alpha>3$ (Lemma 17), each $S \in\left|\mathcal{I}_{C, M}(4)\right|$ is irreducible. Let $\mathcal{B}$ denote the linear system on $W^{\prime}$ induced by $\left|\mathcal{I}_{C, M}(4)\right|$ and fix a general $S \in\left|\mathcal{I}_{C, M}(4)\right|$. Write $S \cap W^{\prime}=C+C^{\prime} \in \mathcal{B}$. Since $C^{\prime}$ is linked to $C$ by the complete intersection $S \cap W^{\prime}$, we have $\operatorname{deg}\left(C^{\prime}\right)=8$ and $p_{a}\left(C^{\prime}\right)=-10$ (19, Proposition $3.1]$ ). Hence $C^{\prime}$ has a multiple component. Since $W^{\prime}$ contains no curve of degree $x \in\{2,3\}$, the multiple component is a line. Since $W^{\prime}$ has a unique line, $R$, $R$ is the multiple component. We saw that $R \cap C=\emptyset$ and $C \subset W_{\text {reg. Since }}$ $\operatorname{dim}(\mathcal{B})>0, \mathcal{B}$ has at least one movable component, $A$. By Lemma 16 is a plane curve of degree $x \in\{4,5\}$. We have $C \cup R \cup A \subset S$. First assume $x=5$. Since $A \in\left|\mathcal{O}_{S}(1)\right|, C \cup R$ is contained in an element of $\left|\mathcal{O}_{S}(3)\right|$. Since the restriction map $H^{0}\left(M, \mathcal{O}_{M}(3)\right) \rightarrow H^{0}\left(S, \mathcal{O}_{S}(3)\right)$ we get $\alpha \leq 3$, contradicting Lemma 17. Now assume $x=4$. Since $R$ is the only line of $W^{\prime}$ we get $A \cup R \in\left|\mathcal{O}_{S}(1)\right|$. As above we get $\alpha \leq 3$, a contradiction.

\section{REFERENCES}

[1] E. Ballico and Ph. Ellia, The maximal rank conjecture for non-special curves in $\mathbf{P}^{3}$, Invent. Math. 79 (1985), 541-555.

[2] E. Ballico and Ph. Ellia, On postulation of curves in $\mathbb{P}^{4}$, Math. Z. 188 (1985), 215-223.

[3] A. Bernardi, A. Gimigliano and M. Idà, Computing symmetric rank for symmetric tensors, J. Symbolic. Comput., 46 (2011), 34-53.

[4] H. Clemens, Some results about Abel-Jacobi mappings. Topics in transcendental algebraic geometry (Princeton, N.J., 1981/1982), 289-304, Ann. of Math. Stud., 106, Princeton Univ. Press, Princeton, NJ, 1984.

[5] E. Cotterill, Rational curves of degree 10 on a general quintic threefold, Comm. Algebra 33 (2005), 1833-1872.

[6] E. Cotterill, Rational curves of degree 11 on a general quintic 3-fold, Quart. J. Math. 63 (2012), 539-568. 
[7] D'Almeida, Courbes rationnelles de degré 11 sur une hypersurface quintique générale de $\mathbb{P}^{4}$, Bull. Sci. math. 136 (2012), 899-903.

[8] D. Eisenbud and J. Harris, Finite projective schemes in linearly general position, J. Algebraic Geom. 1 (1992), 15-30.

[9] Ph. Ellia and Ch. Peskine, Groupes de points de $\mathbf{P}^{2}$ : caractère et position uniforme, in: Algebraic geometry (L' Aquila, 1988), 111-116, Lecture Notes in Math., 1417, Springer, Berlin, 1990.

[10] L. Gruson, R. Lazarsfeld and Ch. Peskine, On a theorem of Castelnuovo, and the equations defining space curves, Invent. Math. 72 (1983), 491-506.

[11] J. Harris, with the collaboration of D. Eisenbud, Curves in Projective Space, Les Presses de l'Université de Montréal, Montréal, 1982.

[12] P. Jahnke, T. Peternell, and I. Radloff, Some recent developments in the classification theory of higher dimensional manifolds, Global aspects of complex geometry, 311-357, Springer, Berlin, 2006.

[13] T. Johnsen and S. Kleiman, Rational curves of degree at most 9 on a general quintic threefold, Comm. Algebra 24 (1996), 2721-2753.

[14] S. Katz, On the finiteness of rational curves on quintic threefolds, Compositio Math. 60 (1986), 151-162.

[15] C. McCrory, T. Shifrin and R. Varley, The Gauss map of a generic hypersurface in $\mathbf{P}^{4}, J$. Differential Geom. 30 (1989), 689-759.

[16] J. Migliore, Geometric invariants for liaison of space curves, J. Algebra 99 (1986), 548-572.

[17] P. G. J. Nijsse, Clemens' conjecture for octic and nonic curves. Indag. Math. 6 (1995), 213221.

[18] D. Perrin, Courbes passant par $m$ points généraux de $\mathbb{P}^{3}$, Bull. Soc. Math. France, Mémoire 28/29 (1987).

[19] C. Peskine and L. Szpiro, Liaison des variétés algébriques. I, Invent. Math. 26 (1974), 271-302.

[20] P. Rao, Liaison among curves in $\mathbb{P}^{3}$, Invent. Math. 50 (1979), 205-217.

[21] R. Strano, A characterization of complete intersection curves in $\mathbf{P}^{3}$, Proc. Amer. Math. Soc. 104 (1998), 711-715.

[22] F. L. Zak, Tangents and secants of algebraic varieties. Translations of Mathematical Monographs, 127. American Mathematical Society, Providence, RI, 1993.

Dipartimento di Matematica, Università di Trento, 38123 Povo (TN), Italy

E-mail address: edoardo.ballico@unitn.it, claudio.fontanari@unitn.it 